\title{
Balance of Autonomic Nervous System Predicts Who Benefits from a Self-management Intervention Program for Irritable Bowel Syndrome
}

\author{
Monica E Jarrett, ${ }^{1}$ Kevin C Cain, ${ }^{2}$ Pamela G Barney, ${ }^{1}$ Robert L Burr, ${ }^{1}$ Bruce D Naliboff, ${ }^{3}$ Robert Shulman, ${ }^{4}$ Jasmine Zia, ${ }^{5}$ and \\ Margaret M Heitkemper ${ }^{1 *}$ \\ ${ }^{1}$ Department of Biobehavioral Nursing and Health Systems, University of Washington, Seattle, WA, USA; ${ }^{2}$ Deptartment of Biostatistics and \\ Office for Nursing Research, University of Washington, Seattle, WA, USA; ${ }^{3}$ Center for Neurovisceral Sciences \& Women's Health, University \\ of California Los Angeles, Los Angeles, CA, USA; ${ }^{4}$ Baylor College of Medicine, Houston, TX, USA; and ${ }^{5}$ Department of Medicine, University of \\ Washington, Seattle, WA, USA
}

\section{Background/Aims}

To determine if potential biomarkers can be used to identify subgroups of people with irritable bowel syndrome (IBS) who will benefit the most or the least from a comprehensive self-management (CSM) intervention.

\section{Methods}

In a two-armed randomized controlled trial a CSM $(n=46)$ was compared to a usual care $(n=46)$ group with follow-up at 3 and 6 months post randomization. Biomarkers obtained at baseline included heart rate variability, salivary cortisol, interleukin-10 produced by unstimulated peripheral blood mononuclear cells, and lactulose/mannitol ratio. Linear mixed models were used to test whether these biomarkers predicted improvements in the primary outcomes including daily abdominal pain, Gastrointestinal Symptom score and IBS-specific quality of life.

\section{Results}

The nurse-delivered 8-session CSM intervention is more effective than usual care in reducing abdominal pain, reducing Gastrointestinal Symptom score, and enhancing quality of life. Participants with lower nighttime high frequency heart rate variability (vagal modulation) and increased low frequency/high frequency ratio (sympathovagal balance) had less benefit from CSM on abdominal pain. Salivary cortisol, IL-10, and lactulose/mannitol ratio were not statistically significant in predicting CSM benefit. Baseline symptom severity interacts with treatment, namely the benefit of CSM is greater in those with higher baseline symptoms.

\section{Conclusions}

Cognitively-focused therapies may be less effective in reducing abdominal pain in IBS patients with higher sympathetic tone. Whether this a centrally-mediated patient characteristic or related to heightened arousal remains to be determined.

(J Neurogastroenterol Motil 2016;22:102-111)

\section{Key Words}

Autonomic nervous system; Interleukin 10; Irritable bowel syndrome; Permeability

Received: April 18, 2015 Revised: July 9, 2015 Accepted: August 4, 2015

(a) This is an Open Access article distributed under the terms of the Creative Commons Attribution Non-Commercial License (http://creativecommons. org/licenses/by-nc/4.0) which permits unrestricted non-commercial use, distribution, and reproduction in any medium, provided the original work is properly cited.

${ }^{*}$ Correspondence: Margaret M Heitkemper, PhD, RN

Department of Biobehavioral Nursing and Health Systems, University of Washington, Box 357266, Seattle, WA 98195-7266, USA Tel: +1-206-543-1091, Fax: +1-206-543-4771, E-mail: heit@uw.edu 


\section{Introduction}

There are increasing researches supporting behavioral and psychological therapies for irritable bowel syndrome (IBS) symptom management. In 2 meta-analyses ${ }^{1,2}$ researchers reported that cognitively-focused therapies such as cognitive behavioral therapy (CBT), do better (ie, reduced gastrointestinal [GI] symptom distress, enhanced quality of life [QOL]) than usual care (UC) or wait-list control groups. However, implementation of $\mathrm{CBT}$ or psychotherapy for IBS is often challenged by access to qualified and trained health care providers and costs. These limitations support identifying patients who are most likely to benefit. For example, are there baseline symptoms or biologic factors that might predict who would benefit the most or the least from the addition of a cognitively-focused therapy?

There are several potential pathophysiological mechanisms that are likely to contribute to IBS symptoms and reduced QOL. These alterations include autonomic nervous system (ANS) imbalance, ${ }^{3}$ hypothalamic-pituitary-adrenal axis (HPA) dysregulation, ${ }^{4,5}$ increased intestinal permeability, ${ }^{6,7}$ and immune dysregulation (ie, decreased tissue interleukin-10 [IL-10] level). ${ }^{8}$ However, to date, no measures based on these mechanisms have proven robust enough to qualify as a valid biomarker for distinguishing patients with IBS from healthy individuals. In addition, it is unknown whether measures such as peripheral cytokine levels (reflection of immune status), intestinal permeability (indicative of a "leaky gut"), and/or heart rate variability (HRV, measure of the variability of the beat-to-beat interval) might help determine who would benefit from psychological therapies. It can be hypothesized that those with greater stress responsiveness (low HRV, high cortisol) may benefit more from approaches that are directed toward cognitions, stress reduction, and/or behavior change. In contrast, those with indicators of peripheral inflammation, immune dysregulation, or epithelial barrier disruption may benefit more from a different approach.

Indicators of ANS dysfunction, that is, low vagal tone and greater sympathetic drive, are more prevalent in individuals with IBS compared to healthy controls. ${ }^{9}$ It is not possible to determine whether the ANS imbalance contributes directly to the pathophysiology or is the outcome of IBS (eg, result of chronic stress and suppression of vagal input). However, it has been shown that physical exercise, which increases HRV, does have positive benefit for IBS patients. ${ }^{10}$ Elevated baseline cortisol levels (an indicator of the HPA) may be linked to conditions of chronic stress, hyperarousal, or conditioned fear such as that experienced by at least a subgroup of IBS patients. However, to date neither indicators of sympathovagal balance or HPA activity have been tested as potential predictors, along with psychological factors, of responsiveness to cognitivelyfocused therapies.

Researchers are also examining intestinal permeability, inflammation and immune function in IBS pathophysiology. Increased intestinal permeability is present in a subset of patients with IBS. ${ }^{11}$ With regard to peripheral levels of cytokines inconsistent findings are noted. Several investigators have reported reduced levels of serum anti-inflammatory cytokine IL-10 $0^{12,13}$ or no difference between those with IBS and controls. ${ }^{14}$

The aim of the current study is to determine whether measures such as nighttime ANS balance, lactulose/mannitol ratio (intestinal permeability measure), and serum IL-10, identified in the literature as potential markers in IBS, are predictive of outcomes with a cognitively-focused behavioral therapy. Based on the literature, we hypothesized that those IBS patients with lower HRV, higher salivary cortisol, and higher IL-10 levels would show greater improvement with an intervention combining CBT, diet counselling, and education.

\section{Materials and Methods}

\section{Trial Design}

Our Nurse-delivered Comprehensive Symptom Management (CSM) intervention, described below, has been shown to be effective in 2 previous randomized trials. ${ }^{15,16}$ This report presents results from a third randomized trial of CSM (conducted 2008-2013), the primary goal of which was to test whether baseline biomarkers can predict treatment effectiveness.

A two-armed randomized controlled trial (RCT) with followup at 3 and 6 months post randomization was used to confirm the efficacy of CSM compared to UC control group in adults with IBS. The protocol for recruitment was similar to that used in 2 previously conducted RCTs. ${ }^{15,16}$ First, participants came to the research office to sign the consent form and return their completed questionnaires. Verbal and written directions were given for completing a symptom diary and collecting urine, saliva, and blood samples. Once the baseline assessment was completed the participant's eligibility was confirmed. If they chose to continue in the study they were randomized to CSM or UC. Those in the treatment group were scheduled for 8 weekly sessions. Follow-up outcome data were obtained from both groups at 3 and 6 months after randomization by a research nurse who was blind to treatment assignment. 
Participants were compensated for their time after each assessment. Biomarkers (blood sample, urine collection for permeability testing, and Holter recording) were performed within a 2-week period prior to randomization. The study was approved by the university institutional review board (2/11/2008) and reviewed annually. The study was registered with ClinicalTrials.gov (NCT00907790).

\section{Participants}

\section{Recruitment and eligibility}

As in our prior 2 trials, potential participants with IBS were recruited through general advertisement (flyers, newspapers, public radio, posters on city busses, and targeted mailings to GI clinic patients) in a metropolitan area in the Pacific Northwest (United States). Interested adults were screened over the phone. Eligibility was assessed across the 5 -week baseline assessment (initial interview and 4-week diary). During the last 2 weeks of this assessment pe- riod, preselected candidate biomarkers were obtained. The criteria for inclusion specified men and women 18-70 years of age. In addition, participants had to have a history of IBS symptoms for at least 6 months prior to their IBS diagnosis and for at least 6 months after their diagnosis by a healthcare provider. They had to meet the Rome-III research criteria. ${ }^{17}$ Adults age 50 or older had to have a negative colonoscopy, sigmoidoscopy, abdominal ultrasonography, or barium enema. Anyone with a "red flag" symptom (eg, lost 10 lbs without trying, blood in stool - except blood due to hemorrhoids) was referred to their healthcare provider for further evaluation (eg, colonoscopy).

Potential participants were excluded if they were taking the following medications: antibiotics, corticosteroids, daily use of anticholinergics, tricyclic antidepressants, calcium-channel blockers; had a medical history of abdominal surgery (except appendectomy, Caesarian section, tubal ligation, laparoscopic cholecystectomy, hysterectomy, or abdominal wall hernia repair); organic GI disease, celiac

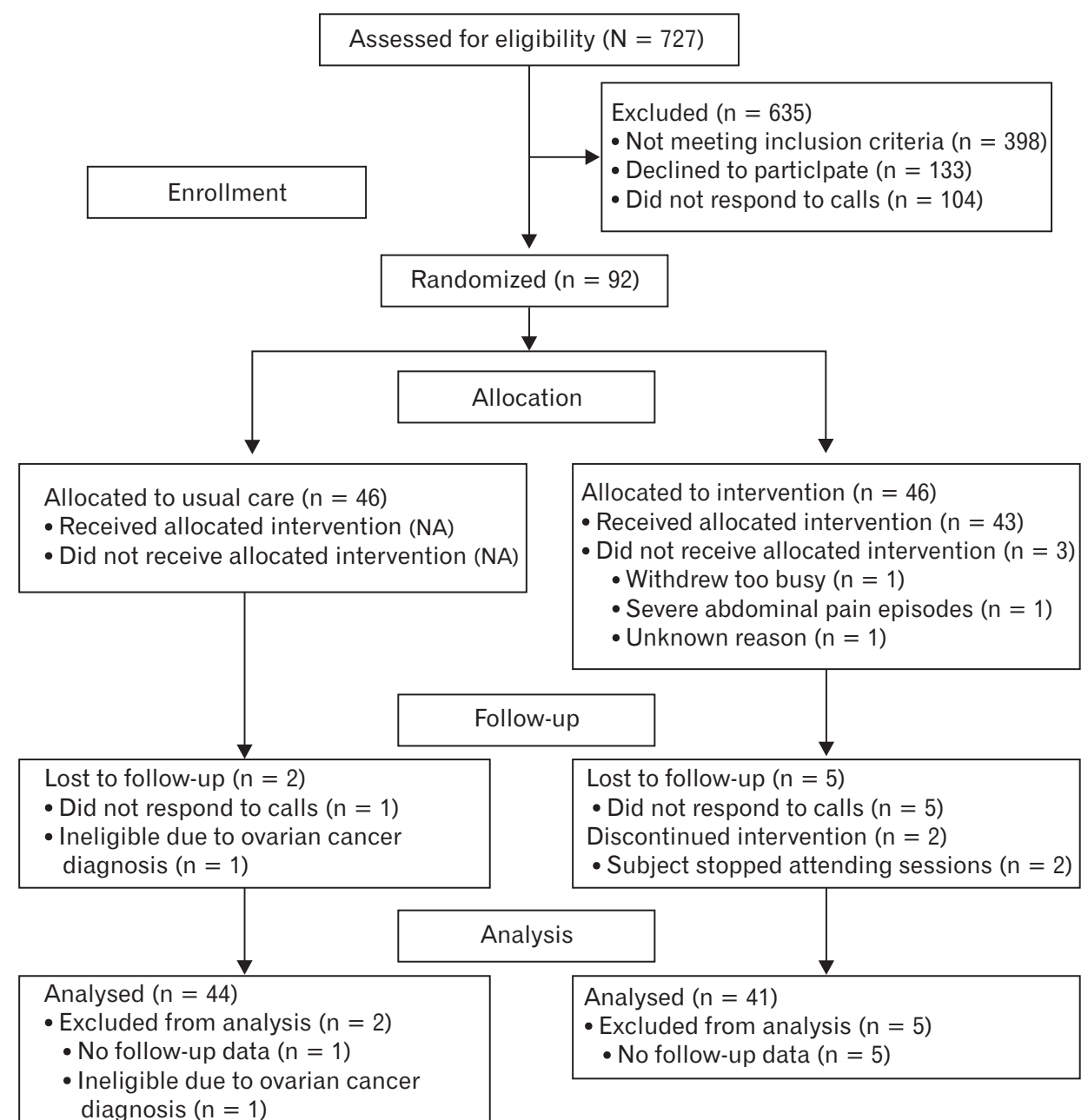

Figure. CONSORT flow diagram identifies the number of participants in each phase of the study. NA, not applicable. 
disease, or a moderate to severe pain condition (eg, low back pain and fibromyalgia); diabetes, current mental health disorders (psychosis, bipolar disorder, or moderate to severe depressive episodes, recent suicide attempt or drug or alcohol abuse or dependence); cardiac valve or conduction defects, immune-compromised disorders (eg, autoimmune conditions) or women who were pregnant, breast feeding, or planning to get pregnant in the next year (Figure).

\section{Interventions}

The CSM intervention was delivered by a research nurse in 8 individual sessions lasting 60 minutes. Participants had 12 weeks to complete the series, thus allowing for personal and family unexpected events. Each person could choose to do all the sessions inperson, over the telephone or a mixture of in-person and telephone. An "IBS Managing Symptoms Workbook" was given to each participant as described in prior studies. ${ }^{15,18}$ The chapters included detailed information on each topic (eg, autogenic relaxation and identifying false beliefs), worksheets, and homework assignments for the following week. Audio tapes of assigned relaxation exercise were included (Supplementary Table 1). Men and women in the UC group were notified that they would not receive the CSM intervention, but should continue with whatever treatment was recommended or provided by their usual healthcare provider. At the end of the study, the participants in the UC group were given the materials used in the CSM intervention.

\section{Primary Outcomes}

\section{Abdominal pain and Gastrointestinal Symptom Score}

GI symptoms (abdominal pain, abdominal distension, bloating, constipation, diarrhea, intestinal gas, and urgency) were rated in a daily diary as not present (0), mild (1), moderate (2), severe (3), or very severe (4), each evening for 4 consecutive weeks. ${ }^{15}$ Abdominal pain was summarized across days as the percent of days with abdominal pain/discomfort that was "moderate" to "very severe." A GI Symptom score was defined as the percent of days that at least one IBS symptom was "moderate" to "very severe."

\section{Quality of life}

QOL was measured with the 42-item IBS quality of life questionnaire (IBSQOL) ${ }^{19}$ The items were grouped into 9 scales: sleep, emotional, mental health beliefs, energy, physical functioning, diet, social role, physical role, and sexual relations. The items from each scale were rated for the past 4 weeks on a 5 or 6 point Likert scale. Extensive and acceptable validity and reliability tests have been con- ducted for the IBSQOL. ${ }^{19}$ A summary QOL score was computed as the average of seven of these scales, excluding the diet scale (since identifying trigger foods as part of CSM may lead to increased food avoidance) and sexual relations scale (since many participants had missing values due to not being sexually active in the prior 4 weeks).

\section{Secondary Outcomes}

\section{Additional diary measures}

Based on the daily diary, the individual GI symptoms of diarrhea, constipation, and urgency were analyzed. Two symptom scales were created: intestinal gas/bloating scale (abdominal distension, bloating, and intestinal gas) and psychological distress scale (anger, anxiety, depressed/sad or blue, and stressed). The symptoms were summarized as the percent of days rated as "moderate" to "very severe." The diary also included a question "How much did your IBS symptoms affect your ability to carry out normal daily activities, other than work?," which was summarized as the percent of days rated as "some," "quite a lot," or "very severe."

\section{Cognitive scale for functional bowel disorders}

Cognitive scale for functional bowel disorders (CSFBD) describes 25 cognitive beliefs related to functional bowel disorders. ${ }^{20}$ The items are rated from 1 "strongly disagree" to 7 "strongly agree," and are summed to make a total score.

\section{Candidate Biomarkers}

\section{Heart rate variability}

Ambulatory electrocardiogram Holter monitors were used to measure nighttime HRV spanning 12 or more hours. Burdick 6732 3-channel compact digital Holter recorders (Burdick Inc, Deerfield, WI, USA) were used, and the resulting records processed using Burdick Vision Premier Analysis software. Trained and crossvalidated operators screened for misclassified artifact, ectopy, and apparent non-sinus rhythms. Standard 4 hour (2-6 AM) nocturnal frequency domain HRV summary measures were computed based on power spectral analysis of R-R intervals in successive 5 minute blocks. ${ }^{21,22}$ Low frequency (LF) band spectral power was defined as the average over 4 hours of the integration of the Fourier HRV spectra between frequencies $\mathrm{f}=0.04$ and $\mathrm{f}=0.15 \mathrm{~Hz}$ (units $=$ milliseconds ${ }^{2}$ ). Both the sympathetic and parasympathetic branches can contribute to the heart period fluctuation components measured in the LF band. The power (variance) in the high frequency (HF) band was estimated by integration of the HRV spectra between fre- 
quencies of $\mathrm{f}=0.15$ and $\mathrm{f}=0.40 \mathrm{~Hz}$. This band power is usually interpreted as an index of parasympathetic (vagal) modulation. The derived measure $\mathrm{LF} / \mathrm{HF}$ ratio is usually interpreted as an index of sympathovagal balance between the branches. A relative increase in sympathetic branch activity, or a relative decrease of parasympathetic activity, or both, will result in an increase in the $\mathrm{LF} / \mathrm{HF}$ ratio index. Since HRV measures are associated with age and body mass index $(\mathrm{BMI}){ }^{23}$ regression equations were fit with $\mathrm{HF}$ power and $\mathrm{LF} / \mathrm{HF}$ ratio as outcomes and age and $\mathrm{BMI}$ as covariates, and the residuals from these regressions were used as the two measures of HRV.

\section{Salivary cortisol}

Saliva was obtained using a salivette (cotton/polypro plug). A radioimmunoassay (RIA) cortisol diagnostic kit was used (Diagnostic Products, Inc, Los Angeles, CA, USA). All assays are performed with duplicate samples and standards. The detection limit is $0.037 \mu \mathrm{g} / \mathrm{dL}$ to $1.0 \mu \mathrm{g} / \mathrm{dL}$ and there is low cross-reactivity with other steroids. Sensitivity range of the kit was $0.003 \mu \mathrm{g} / \mathrm{dL}$ to $2.5 \mu \mathrm{g} / \mathrm{dL}$. The laboratory inter-assay coefficient of variability $(\mathrm{CV})$ was $3.3 \%$; intra-assay $\mathrm{CV}$ was $6.4 \%$.

\section{Interleukin-10 production}

A blood sample was collected in a sodium citrate CPT Vacutainer (Becton Dickinson, Franklin Lakes, NJ, USA). Peripheral blood mononuclear cells (PBMCs) were isolated and incubated for 72 hours. ${ }^{11}$ The supernatant was stored at $-70^{\circ} \mathrm{C}$ until tested for IL-10 using an ELISA assay (R\&D Systems, Minneapolis, MN, USA). Samples were run in duplicate using the manufacturer's instructions. If the $\mathrm{CV}$ for a sample was higher than $15 \%$ the sample was rerun. The minimum detectable concentration for IL-10 was $3.9 \mathrm{pg} / \mathrm{mL}$.

\section{Intestinal permeability testing}

After the evening meal, participants fasted for 4 hours, urinated, then drank a $127.5 \mathrm{~mL}$ solution containing lactulose $(5 \mathrm{~g} / \mathrm{dL})$ and mannitol $(1 \mathrm{~g} / \mathrm{dL})$ followed by $240 \mathrm{~mL}$ water. For the next 24 hours, a plastic "hat" was placed over the toilet seat to capture the participant's urine. Each urine sample was placed into a container containing either thimerosal or chlorhexidine to inhibit bacterial growth. Samples were processed as previously described. ${ }^{11}$ The CV in the assay is $<5 \%$. We defined a subgroup of IBS with increased intestinal permeability as those having a lactulose/mannitol ratio of $>0.015^{11}$

\section{Randomization}

A customized computer program was used for randomization in an adaptive manner that ensures the 2 groups remain fairly balanced with respect to: age, sex, baseline severity of abdominal pain, QOL, predominant stool pattern, and history of post-infectious IBS. The algorithm is a modification of the minimization method proposed by Pocock. ${ }^{24,25}$ Once the research nurse enrolled a participant and the project director entered the balancing variables, the program returned that participant's group assignment. Only the research nurse and project director were aware of the group assignment, the second research nurse who collected outcome data was blind to treatment assignment.

\section{Statistical Methods}

The general approach for the statistical analysis is described in detail below for one of the primary outcomes.

Overall CSM treatment efficacy was tested by fitting a linear mixed model with abdominal pain at 3 and 6 months post-randomization as outcome measures, treatment group (CSM versus UC) as the factor of interest, and age, sex, follow-up occasion, and baseline abdominal pain as covariates. Additional analyses used Analysis of Covariance (ANCOVA) to test the effect of CSM separately at each follow-up time ( 3 and 6 months post-randomization).

For each of the potential biomarkers, participants were divided into two subgroups according to whether the baseline value of the biomarker was above or below a threshold. The thresholds for dichotomization of IL-10 production $(250 \mathrm{pg} / \mathrm{mL})$ and permeability as measured by lactulose/mannitol ratio (0.015) were based on previously published analyses that examined the relationship between baseline biomarker values and baseline symptoms and IBS impact measures. ${ }^{11}$ Since HRV is strongly correlated with age and BMI, thresholds for $\mathrm{HF}$ and $\mathrm{LF} / \mathrm{HF}$ ratio were applied to the residuals from linear regression models using age, sex and BMI to predict the $2 \mathrm{HRV}$ measures. The residuals were initially split into thirds since there was no prior basis for determining thresholds, and then, based on initial analysis, the middle group was combined with one of the other 2 to make a one-third versus two-thirds split of the data. Thresholds for defining subgroups based on salivary cortisol were determined in a similar manner (ie, based on thirds).

For each of the candidate biomarkers a linear mixed model analysis model was fit using abdominal pain at 3 and 6 months postrandomization as outcome measures, and using treatment group, baseline biomarker, age, sex, baseline abdominal pain, and the interaction between treatment group and baseline biomarker as predictor 
variables. The interaction term tests whether treatment effect (ie, mean change in abdominal pain with CSM minus mean change in abdominal pain with UC) is different in the 2 biomarker-defined subgroups.

A potential confounding variable in these analyses is the baseline level of abdominal pain. The efficacy of CSM may well differ according to baseline abdominal pain. For example, if baseline abdominal pain is close to zero for a specific patient, that patient has little room for improvement in abdominal pain and hence one would not expect much benefit from the CSM intervention for the outcome measure of abdominal pain. In contrast, a patient with high baseline abdominal pain does have room for improvement and hence could potentially benefit from CSM. If such an interaction between treatment and baseline abdominal pain exists and if there is also a difference in baseline abdominal pain between the two biomarker-defined groups, this could confound the biomarker analyses. Therefore analyses of biomarker effect on CSM effectiveness also included as a covariate the interaction between baseline abdominal pain and treatment group, to control for this potential confounding.

\section{Results}

As seen in the CONSORT (Figure), 92 participants were randomized and outcome data were available on 85 participants. Demographics and clinical characteristics are described in Table 1. As expected in this randomized trial, most baseline characteristics did not differ between the 2 treatment groups. However, the percentage of Caucasian participants in CSM group (90\%) was greater than that in UC group (65\%). Baseline biomarkers did not vary between the 2 groups at baseline (Table 1). In addition there were no differences in biomarkers based on predominant bowel subgroup (Supplementary Table 2).

\section{Efficacy of Comprehensive Self-management Intervention}

In Table 2, $P$-values for testing CSM versus UC are shown 2 ways: based on ANCOVA separately at 3 and 6 months, and based on linear mixed model analysis which combines data from both outcome time points. We will focus on the combined results here, shown in the next to last column of Table 2. Mean Abdominal Pain and GI Symptom score decreased more $(P=0.010$ and $P<0.001$, respectively) and QOL improved more $(P=0.022)$ in the CSM intervention group than in the UC group. Most of the secondary outcomes also showed CSM having significantly greater improve- ment than UC, with particularly strong effects for CSFBD and the daily intestinal gas/bloating scale. As an alternative to mean change as a measure of treatment effect, Supplementary Table 3 shows the percent of participants (responders) who had at least 50\% improvement in each of the symptoms and symptom scores.

The last column in Table 2 shows the p-value for testing the interaction between treatment assignment and the baseline value of the outcome variable. This interaction is significant at $P<0.05$ for all of the measures based on diary symptoms, meaning that people with higher levels of symptoms at baseline obtain more benefit from CSM than those with low baseline symptoms.

\section{Biomarkers Predict Efficacy}

Table 3 shows results from testing whether baseline biomarker subgroups predict treatment effect of CSM for the three primary outcome measures. Results for secondary outcomes are given in Supplementary Table 3. The interaction beta measures the magnitude of this interaction effect. For example, the first row in Table 3 is for HRV as measured by HF as a biomarker and abdominal pain as the outcome. The coefficient of -19.1 means that among

Table 1. Baseline Demographics, Clinical Characteristics, and Biomarkers of Participants in the Usual Care and Comprehensive Selfmanagement Groups

\begin{tabular}{|c|c|c|}
\hline Variable & $\begin{array}{c}\text { UC } \\
(n=44)\end{array}$ & $\begin{array}{c}\text { CSM } \\
(n=41)\end{array}$ \\
\hline \multicolumn{3}{|l|}{ Demographics } \\
\hline Age (mean [SD], yr) & $37.9(15.9)$ & $40.5(14.6)$ \\
\hline Gender (Female, \% [n]) & $86(38)$ & $90(37)$ \\
\hline Race (White, \% [n]) & $65(28)$ & $90(34)^{\mathrm{a}}$ \\
\hline Married or partnered $(\%[\mathrm{n}])$ & $36(16)$ & $49(20)$ \\
\hline Education (Bachelors or above, $\%[\mathrm{n}]$ ) & $74(32)$ & $73(30)$ \\
\hline Income $(>\$ 60000 / \mathrm{yr},(\%[\mathrm{n}])$ & $42(17)$ & $54(20)$ \\
\hline Professional job (\% [n]) & $31(13)$ & $45(18)$ \\
\hline \multicolumn{3}{|l|}{ Predominant bowel pattern $(\%[\mathrm{n}])$} \\
\hline Un-subtyped & $7(3)$ & $3(1)$ \\
\hline Constipation & $12(5)$ & $16(6)$ \\
\hline Diarrhea & $31(13)$ & $37(14)$ \\
\hline Mixed & $50(21)$ & $45(17)$ \\
\hline \multicolumn{3}{|l|}{ Biomarkers (mean [SD]) } \\
\hline High frequency & $727.1(800.9)$ & $614.6(887.5)$ \\
\hline Low frequency/high frequency & $3.86(2.22)$ & $4.51(2.41)$ \\
\hline Lactulose/mannitol ratio & $0.011(0.006)$ & $0.011(0.009)$ \\
\hline Cortisol & $0.33(0.14)$ & $0.32(0.15)$ \\
\hline IL-10 production & $162.9(196.0)$ & $197.1(224.1)$ \\
\hline
\end{tabular}


Table 2. Baseline and Change Scores on the Primary and Secondary Outcome Variables by Usual Care or Comprehensive Self-management

\begin{tabular}{|c|c|c|c|c|c|c|}
\hline & \multirow{2}{*}{$\begin{array}{c}\text { Mean (SD) } \\
\text { Baseline } \\
(n=44,41)\end{array}$} & \multicolumn{2}{|c|}{$\begin{array}{c}\text { Mean }(\mathrm{SD}) \\
\text { Change from Baseline }\end{array}$} & \multicolumn{3}{|c|}{$P$-values ${ }^{c}$} \\
\hline & & $\begin{array}{c}\text { 3-months } \\
(\mathrm{n}=43,40)\end{array}$ & $\begin{array}{c}\text { 6-months } \\
(\mathrm{n}=41,41)\end{array}$ & $\begin{array}{l}\mathrm{CSM}_{\text {vs UC }} \mathrm{UC}^{\mathrm{a}} \\
\text { at } 3 \mathrm{Mo} / 6 \mathrm{Mo}\end{array}$ & $\begin{array}{l}\text { CSM vs } \mathrm{UC}^{\mathrm{b}} \\
\text { at both times }\end{array}$ & $\begin{array}{c}\text { Interaction } \\
\text { Tx by baseline }\end{array}$ \\
\hline \multicolumn{7}{|c|}{ Primary outcomes } \\
\hline \multicolumn{7}{|c|}{ Abdominal pain/discomfort } \\
\hline UC & $37.2(24.7)$ & $-11.1(21.9)$ & $-7.4(22.1)$ & $0.070 / 0.002$ & 0.010 & 0.046 \\
\hline $\mathrm{CSM}$ & $38.8(28.5)$ & $-20.9(25.2)$ & $-22.4(25.8)$ & & & \\
\hline \multicolumn{7}{|c|}{ IBS Symptom score } \\
\hline UC & $62.8(25.2)$ & $-10.0(21.5)$ & $-11.5(20.5)$ & $<0.001 /<0.001$ & $<0.001$ & 0.007 \\
\hline CSM & $65.2(30.4)$ & $-30.4(25.5)$ & $-34.2(28.5)$ & & & \\
\hline \multicolumn{7}{|c|}{ Quality of life } \\
\hline UC & $71.0(14.9)$ & $4.7(11.6)$ & $5.1(11.5)$ & $0.030 / 0.051$ & 0.022 & 0.299 \\
\hline CSM & $69.6(13.5)$ & $10.8(13.1)$ & $11.4(15.3)$ & & & \\
\hline \multicolumn{7}{|c|}{ Secondary outcomes } \\
\hline \multicolumn{7}{|c|}{ CSFBD } \\
\hline UC & $4.5(0.95)$ & $-0.39(0.69)$ & $-0.8(0.87)$ & $<0.001 /<0.001$ & 0.002 & 0.069 \\
\hline CSM & $4.6(1.0)$ & $-0.85(1.0)$ & $-1.1(1.0)$ & & & \\
\hline \multicolumn{7}{|c|}{ IBS interference with activities } \\
\hline UC & $36.9(28.3)$ & $-3.5(25.2)$ & $-2.4(23.9)$ & $0.041 / 0.004$ & 0.007 & 0.226 \\
\hline $\mathrm{CSM}$ & $49.1(30.2)$ & $-19.2(27.9)$ & $-21.8(28.9)$ & & & \\
\hline \multicolumn{7}{|c|}{ Psychological distress } \\
\hline UC & $11.3(12.8)$ & $0.94(11.7)$ & $1.7(12.7)$ & $0.046 / 0.007$ & 0.016 & 0.002 \\
\hline $\mathrm{CSM}$ & $11.8(9.7)$ & $-3.7(7.9)$ & $-5.3(9.6)$ & & & \\
\hline \multicolumn{7}{|c|}{ Bloating/gas } \\
\hline UC & $33.1(25.7)$ & $-8.8(16.0)$ & $-9.3(19.3)$ & $0.001 / 0.001$ & $<0.001$ & $<0.001$ \\
\hline CSM & $36.6(26.7)$ & $-23.5(23.4)$ & $-23.9(23.3)$ & & & \\
\hline \multicolumn{7}{|c|}{ Constipation } \\
\hline UC & $17.4(21.5)$ & $-3.8(20.0)$ & $-5.6(19.2)$ & $0.012 / 0.025$ & 0.011 & 0.025 \\
\hline CSM & $21.5(22.8)$ & $-15.0(18.7)$ & $-15.0(21.2)$ & & & \\
\hline \multicolumn{7}{|l|}{ Diarrhea } \\
\hline UC & $12.1(13.9)$ & $-1.3(11.4)$ & $-0.19(14.8)$ & $0.187 / 0.074$ & 0.113 & 0.030 \\
\hline $\mathrm{CSM}$ & $18.0(24.9)$ & $-9.2(21.4)$ & $-9.8(22.2)$ & & & \\
\hline \multicolumn{7}{|l|}{ Urgency } \\
\hline UC & $14.4(19.6)$ & $0.61(17.4)$ & $2.6(8.7)$ & $0.017 /<0.001$ & $<0.001$ & $<0.001$ \\
\hline CSM & $21.0(28.2)$ & $-12.8(23.9)$ & $-15.2(25.8)$ & & & \\
\hline
\end{tabular}

${ }^{\mathrm{a}}$ The first column of $P$-values are based on ANCOVA, controlling for baseline value of the outcome variable, for testing whether the mean outcome differs between the CSM and $\mathrm{UC}$ at 3 and 6 months post randomization, respectively.

${ }^{\mathrm{b}}$ The second column of $P$-values are based on linear mixed model, controlling for baseline value of the outcome variable, for testing whether the mean outcome differs between CSM and UC across both follow-up times.

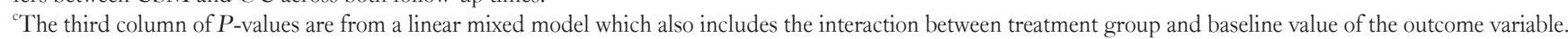
UC, usual care; CSM, comprehensive self-management; IBS, irritable bowel syndrome; CSFBD, cognitive scale for functional bowel disorders.

Other outcomes are based on a 4-week daily diary. IBS Symptom score is defined as the percent of days that at least one IBS symptom (abdominal pain/discomfort, abdominal distension, bloating, intestinal gas, constipation, diarrhea, and urgency) is present with moderate or worse severity. Each individual IBS symptom is summarized for each person as the percent of days that the symptom is present with moderate or worse severity. The Bloating/Gas scale was made by combining abdominal distension, bloating, intestinal gas and passing gas/flatulence; The Psychological Distress scale was made by combining anxiety, anger, decreased desire to talk or move, depressed/sad or blue, panic feelings, and stressed; and IBS interference with activities is summarized as the percent of days that IBS interferes with daily activities other than work.

those with higher HF, the treatment effect of CSM, defined as mean change in abdominal pain for CSM minus mean change for UC, is 19.1 points lower than for those with lower HF. This nega- tive coefficient indicates that participants with low $\mathrm{HF}$ benefit less from the CSM intervention than do people with higher $\mathrm{HF}(P=$ 0.015). The strongest results in Table 3 are for $\mathrm{HF}$ and $\mathrm{LF} / \mathrm{HF}$, as 
Table 3. Interaction of Intervention Group (Comprehensive Selfmanagement Versus Usual Care) with Baseline Biomarkers for Primary Outcome Variables

\begin{tabular}{|c|c|c|}
\hline Outcomes & Interaction beta $(\mathrm{CI})^{\mathrm{a}}$ & $P$-value ${ }^{\mathrm{b}}$ \\
\hline \multicolumn{3}{|l|}{ HRV-HF $(\mathrm{n}=29 / 56)$} \\
\hline Abdominal pain/discomfort & $-19.1(-34.3,-3.9)$ & 0.015 \\
\hline GI Symptom score & $-12.0(-30.3,6.2)$ & 0.192 \\
\hline Quality of life & $8.5(-1.4,18.4)$ & 0.092 \\
\hline \multicolumn{3}{|l|}{ HRV-LF/HF ratio $(\mathrm{n}=57 / 28)$} \\
\hline Abdominal pain/discomfort & $28.5(14.0,43.1)$ & $<0.001$ \\
\hline GI Symptom score & $19.6(1.9,37.3)$ & 0.030 \\
\hline Quality of life & $-6.0(-16.1,4.1)$ & 0.243 \\
\hline \multicolumn{3}{|l|}{ Salivary cortisol $(\mathrm{n}=50 / 24)$} \\
\hline Abdominal pain/discomfort & $16.4(-0.9,33.7)$ & 0.063 \\
\hline GI Symptom score & $8.0(-12.4,28.4)$ & 0.438 \\
\hline Quality of life & $6.1(-4.9,17.2)$ & 0.271 \\
\hline \multicolumn{3}{|l|}{ IL-10 (n = 60/20) } \\
\hline Abdominal pain/discomfort & $-9.7(-27.8,8.5)$ & 0.291 \\
\hline GI Symptom score & $-0.3(-21.1,21.8)$ & 0.975 \\
\hline Quality of life & $-0.9(-13.7,11.9)$ & 0.893 \\
\hline \multicolumn{3}{|c|}{ Intestinal permeability $(\mathrm{n}=54 / 21)$} \\
\hline Abdominal pain/discomfort & $2.4(-16.0,20.1)$ & 0.815 \\
\hline GI Symptom score & $-0.8(-21.4,19.8)$ & 0.941 \\
\hline Quality of life & $-4.7(-16.7,7.3)$ & 0.439 \\
\hline
\end{tabular}

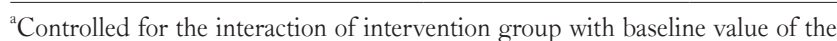
outcome variable. The $2 \mathrm{~N}$ values are the number of participants with lower versus higher biomarker values. Interaction beta measures the extent to which the treatment effect (mean change in comprehensive self-management group minus mean change in usual care group) is stronger in those with higher biomarker versus those with lower biomarker.

${ }^{\mathrm{b}} \mathrm{P}$-value for the interaction between treatment group and biomarker, from a linear mixed model using data from both follow-up times and controlling for baseline of the outcome variable and for the interaction of intervention group with baseline value of the outcome variable. Analyses for HRV also control for age, gender, and body mass index.

HRV-HF, heart rate variability-high frequency; HRV-LF/HF, heart rate variability-low frequency/high frequency; GI, gastrointestinal; IL-10, interleukin-10.

measures of HRV, with Abdominal Pain and GI Symptom score as outcomes. For example among participants with high (top third) $\mathrm{LF} / \mathrm{HF}$ ratio, there is no evidence that CSM is better than UC. But among those in the lower two thirds of $\mathrm{LF} / \mathrm{HF}$ there is strong evidence that CSM is better than UC.

Although there was only a trend in the results for salivary cortisol $(P=0.063$; Table 3$)$ the beta values are consistent with the HRV results, namely that the treatment effect is weaker among those with higher cortisol. In addition, those with higher cortisol levels showed less CSM effect on psychological distress $(P=0.014$; Supplementary Table 4). IL-10 shows a similar non-significant pattern $(P=0.085)$ with CSM efficacy being higher in those with higher IL-10. The lactulose/mannitol ratio levels did not predict who would benefit from CSM relative to UC.

\section{Discussion}

As previously demonstrated in separate samples, ${ }^{15,16}$ the nursedelivered 8-session CSM intervention is more effective than UC in reducing abdominal pain, GI Symptom score and enhancing QOL (primary outcomes) as well as reducing most other GI symptoms (bloating/gas, constipation and urgency), negative cognitive beliefs about IBS, IBS interference with activities, and psychological distress (secondary outcomes). By including measures of ANS balance, IL-10, and permeability, the current results extend our prior work as well as others who have demonstrated positive outcomes following cognitively-focused behavioral therapies. Contrary to our original hypothesis, IBS participants with lower nighttime vagal modulation and higher sympathovagal balance had less benefit from CSM on the primary outcome of abdominal pain. In addition we found that there was a trend for less benefit from CSM among those with higher salivary cortisol levels and a weak trend for more benefit from CSM among those with higher production of IL-10 by unstimulated PBMCs. In contrast, the lactulose/mannitol ratio did not predict benefit from CSM. As shown by others, baseline symptom severity interacts with treatment; namely the benefit of CSM is greater in those with higher baseline symptoms.

Ours is the first study to suggest that HRV may be related to the likelihood of not benefitting (no change in abdominal pain and GI Symptom score) from cognitively-focused behavioral therapies. It has been suggested that low HRV-HF and high HRV-LF/HF ratio are indicative of an inflexible ANS, which places the individual at risk for poor health. ${ }^{26,27}$ Higher night-time sympathovagal balance and perhaps heightened HPA activation may reflect a reduced capacity to develop and retain new strategies (relaxation, cognitive restructuring) for symptom management and as such, may signify those individuals less likely to benefit from $\mathrm{CSM} .{ }^{27}$ Consistent with this hypothesis we found some evidence that IBS patients with higher levels of salivary cortisol were less likely to benefit from CSM on the primary outcome measure of abdominal pain and two secondary outcomes, ie, psychological distress and CSFBD.

We observed a weak trend of higher IL-10 production by unstimulated PBMCs being associated with increased responsiveness to CSM. IL-10 is an anti-inflammatory cytokine and contributes to the modulation of a pro-inflammatory response as well as pain sensitivity. ${ }^{11,28,29}$ However, the role of anti-inflammatory cytokines in IBS remains controversial. In a recent systematic review and meta 
analyses of 6 studies, Bashashati et $\mathrm{al}^{30}$ did not find serum IL-10 level differences between IBS and healthy controls.

Contrary to our hypotheses, intestinal lactulose/mannitol ratio levels were not associated with improvements in the primary or secondary outcomes. Combined these analyses suggest that even those who may have evidence of intestinal barrier dysfunction and inflammation improve with the cognitively-focused behavioral intervention.

Those participants with low symptom severity at baseline were unlikely to decrease that symptom, regardless of treatment group. This finding may reflect the presence of a "floor" effect, that is, when symptom severity/frequency was low at baseline there was little room for improvement. IBS is a heterogeneous condition, not only because of different bowel pattern predominance (the majority in the current study were diarrhea predominant or mixed), but also with regard to symptom severity and frequency. ${ }^{31,32}$ An eligibility criterion for enrollment was abdominal pain/discomfort ("mild" to "severe") at least 2 days per week during the baseline assessment. While all participants met this criterion in terms of pain frequency, for some participants the abdominal pain/discomfort severity was often rated as "mild." Other symptoms such as bloating were not included in the eligibility criteria and hence some participants with low frequency of those symptoms were included in the study.

There are several limitations of the current study. First, the modest sample size means power for detecting interaction effects is low unless the effect is quite strong. While some biomarkers show strong interactions that are significant, others show weaker effects that are not significantly different from zero but the wide confidence interval means that the data are consistent with a moderately large effect. Second, race varied by treatment group. However, a post-hoc analyses of both primary and secondary outcomes while controlling for race failed to alter the conclusions. Third, the CSM intervention is multi-component, thus it is not possible to discriminate which element(s) were most likely to produce the overall positive benefit of the treatment. Fourth, because this was a behavioral intervention trial it was not possible to "blind" participants to the treatment they were receiving. Finally, multiple comparisons were made and thus, the findings should be interpreted with caution.

In summary, our results add to the growing body of literature that supports the incorporation of cognitively-focused therapy for IBS symptom management. Unfortunately, CSM is not readily accessible to everybody despite its demonstrated effectiveness. The current study extends our understanding of the characteristics of those individuals who are less likely to benefit from CSM. Further research into the clinical utility of using measures of ANS to identify those individuals with high potential for benefitting from cognitively-focused therapies may be warranted. This could eventually help clinicians focus behavioral therapies for IBS to those most likely to benefit.

\section{Supplementary Materials}

Note: To access the supplementary tables mentioned in this article, visit the online version of Journal of Neurogastroenterology and Motility at http://www.jnmjournal.org/, and at http://dx.doi. org/10.5056/jnm15067.

Financial support: This research was supported by National Institute of Nursing Research, National Institutes of Health, USA (Grant No. NR004142).

\section{Conflicts of interest: None.}

Author contributions: Margaret M Heitkemper, Monica E Jarrett, Kevin C Cain, and Robert L Burr planned the study; Pamela G Barney and Monica E Jarrett conducted the study and collected data; Margaret M Heitkemper, Monica E Jarrett, Kevin C Cain, Robert L Burr, and Robert Shulman analyzed and interpreted the data; and Margaret M Heitkemper, Monica E Jarrett, Kevin C Cain, Robert L Burr, Bruce D Naliboff, and Jasmine Zia conducted drafting and revision of the manuscript.

\section{References}

1. Lackner JM, Mesmer C, Morley S, Dowzer C, Hamilton S. Psychological treatments for irritable bowel syndrome: a systematic review and meta-analysis. J Consult Clin Psychol 2004;72:1100-1113.

2. Zijdenbos IL, de Wit NJ, van der Heijden GJ, Rubin G, Quartero AO. Psychological treatments for the management of irritable bowel syndrome. Cochrane Database Syst Rev 2009:Cd006442.

3. Cheng P, Shih W, Alberto M, et al. Autonomic response to a visceral stressor is dysregulated in irritable bowel syndrome and correlates with duration of disease. Neurogastroenterol Motil 2013;25:e650-e659.

4. Heitkemper MM, Cain KC, Deechakawan W, et al. Anticipation of public speaking and sleep and the hypothalamic-pituitary-adrenal axis in women with irritable bowel syndrome. Neurogastroenterol Motil 2012; 24:626-631, e270-e271.

5. Chang L, Sundaresh S, Elliott J, et al. Dysregulation of the hypothalamic-pituitary-adrenal (HPA) axis in irritable bowel syndrome. Neurogastroenterol Motil 2009;21:149-159.

6. Wilcz-Villega E, McClean S, O'Sullivan M. Reduced E-cadherin expression is associated with abdominal pain and symptom duration in a study of alternating and diarrhea predominant IBS. Neurogastroenterol 
Motil 2014;26:316-325.

7. Piche T, Barbara G, Aubert P, et al. Impaired intestinal barrier integrity in the colon of patients with irritable bowel syndrome: involvement of soluble mediators. Gut 2009;58:196-201.

8. Chang L, Adeyemo M, Karagiannides I, et al. Serum and colonic mucosal immune markers in irritable bowel syndrome. Am J Gastroenterol 2012;107:262-272.

9. Burr RL, Heitkemper M, Jarrett M, Cain KC. Comparison of autonomic nervous system indices based on abdominal pain reports in women with irritable bowel syndrome. Biol Res Nurs 2000;2:97-106.

10. Johannesson E, Simrén M, Strid H, Bajor A, Sadik R. Physical activity improves symptoms in irritable bowel syndrome: a randomized controlled trial. Am J Gastroenterol 2011;106:915-922.

11. Shulman RJ, Jarrett ME, Cain KC, Broussard EK, Heitkemper MM. Associations among gut permeability, inflammatory markers, and symptoms in patients with irritable bowel syndrome. J Gastroenterol 2014; 49:1467-1476.

12. Schmulson M, Pulido-London D, Rodriguez O, et al. Lower serum IL10 is an independent predictor of IBS among volunteers in Mexico. Am J Gastroenterol 2012;107:747-753.

13. Darkoh C, Comer L, Zewdie G, Harold S, Snyder N, Dupont HL. Chemotactic chemokines are important in the pathogenesis of irritable bowel syndrome. PLoS One 2014;9:e93144.

14. Rana SV, Sharma S, Sinha SK, Parsad KK, Malik A, Singh K. Proinflammatory and anti-inflammatory cytokine response in diarrhoea-predominant irritable bowel syndrome patients. Trop Gastroenterol 2012;33: 251-256.

15. Jarrett ME, Cain KC, Burr RL, Hertig VL, Rosen SN, Heitkemper MM. Comprehensive self-management for irritable bowel syndrome: randomized trial of in-person vs. combined in-person and telephone sessions. Am J Gastroenterol 2009;104:3004-3014.

16. Heitkemper MM, Jarrett ME, Levy RL, et al. Self-management for women with irritable bowel syndrome. Clin Gastroenterol Hepatol 2004;2:585-596.

17. Drossman D, Corazziari E, Delvaux M, et al, ed. Rome III: The functional gastrointestinal disorders. 3rd ed. McLean,VA: Degnon Associates, Inc. 2006:49.

18. Barney P, Weisman P, Jarrett M, Levy R, Heitkemper M. Master your IBS: an 8-week plan proven to control the symptoms of irritable bowel syndrome. Bethesda, MD: AGA Press 2010: 1-229.

19. Hahn BA, Kirchdoerfer LJ, Fullerton S, Mayer E. Evaluation of a new quality of life questionnaire for patients with irritable bowel syndrome.
Aliment Pharmacol Ther 1997;11:547-552.

20. Toner BB, Stuckless N, Ali A, Downie F, Emmott S, Akman D. The development of a cognitive scale for functional bowel disorders. Psychosom Med 1998;60:492-497.

21. Task Force of the European Society of Cardiology and the North American Society of Pacing and Electrophysiology. Heart rate variability: standards of measurement, physiological interpretation and clinical use. Circulation 1996;93:1043-1065.

22. Kleiger RE, Stein PK, Bigger JT Jr. Heart rate variability: measurement and clinical utility. Ann Noninvasive Electrocardiol 2005;10:88-101.

23. Umetani K, Singer DH, McCraty R, Atkinson M. Twenty-four hour time domain heart rate variability and heart rate: relations to age and gender over nine decades. J Am Coll Cardiol 1998;31:593-601.

24. Pocock SJ, Simon R. Sequential treatment assignment with balancing for prognostic factors in the controlled clinical trial. Biometrics 1975;31:103115 .

25. Pocock S. Clinical trials - a practical approach. Chichester: John Wiley \& Sons 1983:80-87.

26. Carnevali L, Sgoifo A. Vagal modulation of resting heart rate in rats: the role of stress, psychosocial factors, and physical exercise. Front Physiol 2014;5:118.

27. Pittig A, Arch JJ, Lam CW, Craske MG. Heart rate and heart rate variability in panic, social anxiety, obsessive-compulsive, and generalized anxiety disorders at baseline and in response to relaxation and hyperventilation. Int J Psychophysiol 2013;87:19-27.

28. Willemen HL, Eijkelkamp N, Garza Carbajal A, et al. Monocytes/ macrophages control resolution of transient inflammatory pain. J Pain 2014;15:496-506.

29. Hughes PA, Harrington AM, Castro J, et al. Sensory neuro-immune interactions differ between irritable bowel syndrome subtypes. Gut 2013; 62:1456-1465.

30. Bashashati M, Rezaei N, Shafieyoun A, et al. Cytokine imbalance in irritable bowel syndrome: a systematic review and meta-analysis. Neurogastroenterol Motil 2014;26:1036-1048.

31. Ford AC, Bercik P, Morgan DG, Bolino C, Pintos-Sanchez MI, Moayyedi P. Characteristics of functional bowel disorder patients: a crosssectional survey using the Rome III criteria. Aliment Pharmacol Ther 2014;39:312-321.

32. Gerson CD, Gerson J, Gerson MJ. Group hypnotherapy for irritable bowel syndrome with long-term follow-up. Int J Clin Exp Hypn 2013; 61:38-54. 